Improved student learning in ophthalmology with computer-aided instruction

\begin{abstract}
Purpose Recent changes in the medical school curriculum and in teaching styles have stimulated the development of novel learning resources. We investigated the impact of computer-aided learning in the field of ophthalmology at a large Australian undergraduate medical school.

Methods Clinical case studies describing common ophthalmic conditions were incorporated into a computing program which uses a problem-solving format. The program was made available to 85 medical students for a 2 week period. A control group of 20 medical students did not have access to this material. Acquisition of new knowledge was assessed as the improvement in score on a knowledgebased multiple-choice examination which students took both before and following the learning interval.

Results Students in the control group showed no improvement over the test period, whilst the students who had access to the computer significantly increased their knowledge base, both for material provided on the program $(p=0.0001)$ and in related areas $(p=0.0015)$. Conclusions Suitably constructed material on the computer is a useful aid in teaching ophthalmology to medical undergraduates. The material and the medium may also act as a stimulus for further learning.
\end{abstract}

Key words Computer, Medical school curriculum, Ophthalmology, Teaching

Increasing numbers of medical schools worldwide are successfully adopting the problem-based learning style originally popularised at McMaster University. ${ }^{1-4}$ The underlying philosophy of this form of teaching is that active student participation in their own education will encourage a deeper, clearer understanding and nurture the discipline of self-directed learning. ${ }^{5}$ In other words, giving students problems to actively consider and unravel is a more effective mode of learning than the passive delivery of a lecture-based
PETER DEVITT, JUSTINE R. SMITH,

EDWARD PALMER course. This shift in educational method is particularly important for ophthalmology, given the strong, unfortunately legitimate, criticisms of traditional methods used to teach this medical specialty. ${ }^{6,7}$ One recent study has demonstrated significant benefits of problembased learning for the teaching of ophthalmology, in terms of large knowledge gains and high levels of student satisfaction. ${ }^{8}$

Whilst advances have been made in educational techniques, today's medical students face new difficulties. As a direct effect of cost-cutting policies, such as outpatient and day case procedures, day-of-surgery admissions and managed care, these students have reduced access to patients. ${ }^{9}$ Furthermore, increasing emphasis is being placed on teaching in the primary care centres, and major teaching institutions now play a less pivotal role in medical education than previously. With more time spent at locations remote from the main teaching centre, it is difficult to timetable a curriculum in which clinical exposure coincides with the formal teaching of a particular discipline.

Computer-aided learning has several key features which may overcome these problems. This resource can be made readily available at any time of the year and anywhere students are based, to complement the clinical experience. In addition, the content can be constructed and/or periodically reviewed and updated by the appropriate experts. At the University of Adelaide, a problem-based learning computer software product termed Medici was recently developed. The program has been used to provide supplementary learning materials for students on clinical attachments, ${ }^{10}$ and it has been shown to be as effective as tutorials for the delivery of educational material. ${ }^{11}$ Traditionally students had been instructed in ophthalmology primarily by lectures, but, in 1998, Medici became the predominant means of teaching this specialty. We undertook a study to formally evaluate this ophthalmic teaching resource.
P. Devitt

Department of Surgery University of Adelaide Adelaide, Australia

\section{J.R. Smith}

Department of

Ophthalmology

Flinders University of South

Australia

Adelaide, Australia

E. Palmer

Department of Medical

Education

University of Adelaide

Adelaide, Australia

Peter Devitt

Department of Surgery University of Adelaide

Royal Adelaide Hospital North Terrace

Adelaide, South Australia 5000

Australia

Tel: +61 882225144

Fax: +61 882225896

e-mail:

peter.devitt@adelaide.edu.au

Received: 26 September 2000

Accepted in revised form: 6 March 2001 


\section{Materials and methods}

Medici software product

Medici is a Macintosh-compatible, IBM-compatible software package produced in the Department of Medical Education at the University of Adelaide. The computer material presented on Medici takes the form of case scenarios, and encourages students to 'manage' clinical cases in a realistic manner. As decisions are made, Medici moves forward in the management of the case, providing feedback on the correctness of the selections. In this way, students learn from their decision-making as they proceed. The user selects a case, and is presented with history and examination findings, in the form of text, photographs or video images. Subsequently, a number of diagnostic options are offered. After the user makes a diagnosis, additional clinical information is provided, and questions concerning treatment are posed. Each case study culminates in a critique of the problem, and a final score with comment indicates to the student their level of competence in managing the case. An easy-to-use authoring tool allows instructors with limited computing skills to construct and edit clinical cases for the program. Depending on the author's objectives, cases can concentrate on aspects of diagnosis, investigation or treatment. Medici also charts a student's progress through each case, giving the instructor an opportunity to follow student learning and to measure the effectiveness of different case studies in instructing students.

Ophthalmologists from university-affiliated hospitals in South Australia designed 21 ophthalmic cases for Medici. Some cases were brief and depended largely on visual identification of an ocular problem. Other studies were complex and invited the user to advance through several stages of management decision-making. In general, cases were based on real patient encounters that a medical practitioner might expect to manage in everyday general practice or the emergency department of a general hospital. Topics included acute conjunctivitis, cataract, age-related macular degeneration and ocular trauma. Fig. 1 shows the example of a patient presenting with a 'painful red eye' due to viral conjunctivitis.

\section{Student subjects and tuition in ophthalmology}

The University of Adelaide Medical School has a 6 year undergraduate curriculum consisting of a 3 year basic science course followed by a 3 year clinical instruction. The school enrols approximately 600 students each year, the majority of whom are resident in metropolitan and rural South Australia, with small numbers coming from interstate and overseas. This study was undertaken in 1998, during the first 2 weeks of the final year of the medical course, when students received their instruction in ophthalmology. The tuition in ophthalmology consisted of two lectures (an introductory overview of ophthalmology and a lecture on paediatric eye disease, a topic which was not covered by the computer material), a single 3 hour visit to the ophthalmic outpatient department (an instruction in ophthalmic history-taking and clinical examination) and free access to the Medici computer material. Students studying at Flinders University of South Australia Medical School, the second medical school in South Australia, at an equivalent stage in their medical course, acted as a control group. The programs at the two universities run along similar educational lines and endorse the problem-based learning approach. These students did not have access to the Medici software product during the study period.

\section{Enrolment of students into the study}

All the students (in equivalent years) at both universities were invited to participate in the study (110 at the University of Adelaide and 30 at Flinders University). The Flinders University students were to form the control group. The rationale behind this was that this was the only realistic method of isolating a control group who would not access the study material (e.g. by sharing passwords). Before the study started, potential recruits were given the study protocol and the system of pre- and post-testing was explained.

\section{Assessment of student learning}

Students using the Medici software product and control students were examined at the beginning and end of the study period for an increase in knowledge of ophthalmology. The testing material was knowledgebased, and consisted of a written paper with 36 longstemmed multiple-choice questions. Half the questions were based on material contained in the computer case studies, and the other half had no relevance to this material. Each student used a unique password to gain access to Medici, allowing activity on the computer to be recorded, in terms of cases studied and time spent. The students were also informally monitored in the computer suite during three 2 hour study periods.

\section{Statistical analysis}

The difference between pre- and post-tuition test results was calculated for matched pairs within the control and computer groups. Student's $t$-test was used to measure the significance of the difference within these groups. To determine whether there was a significant difference in the level of improvement between the two groups, Student's $t$-test was used, or where the variance of scores within groups was unequal, a standard test of covariance was applied. A statistically significant difference was defined as one giving a $p$ value less than or equal to 0.05 . 

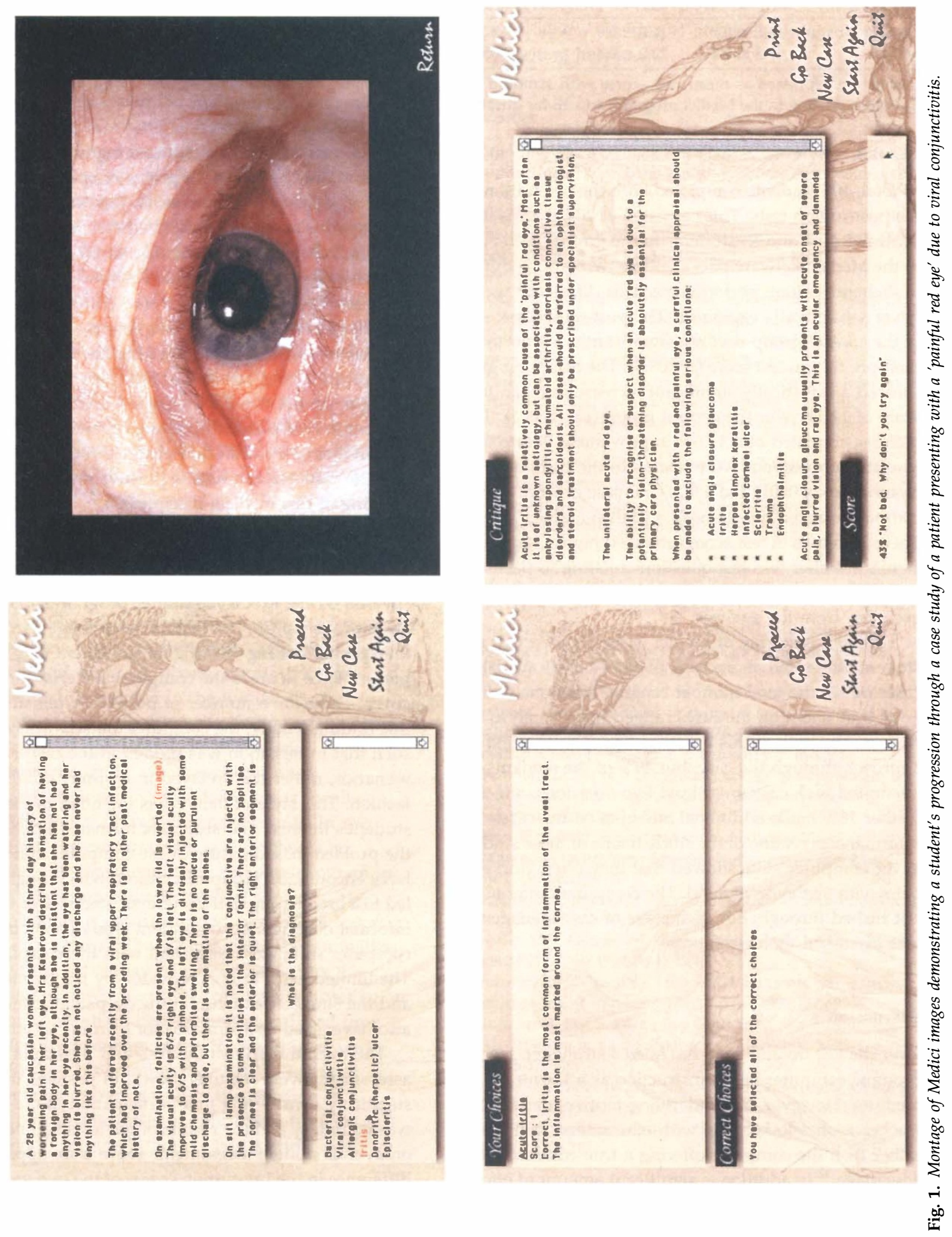
Table 1. Pre-and post-tuition test scores for students given access to the Medici software package and for control students who did not use the program

\begin{tabular}{|c|c|c|c|c|c|c|}
\hline \multirow[b]{2}{*}{ Student group } & \multicolumn{2}{|c|}{ Total score: all questions } & \multicolumn{2}{|c|}{ Specific score: Medici-related questions } & \multicolumn{2}{|c|}{ Specific score: non-related questions } \\
\hline & Pre-tuition & Post-tuition & Pre-tuition & Post-tuition & Pre-tuition & Post-tuition \\
\hline Control & $16.0 \pm 0.7$ & $16.2 \pm 0.9$ & $7.5 \pm 0.5$ & $7.8 \pm 0.5$ & $8.5 \pm 0.5$ & $8.4 \pm 0.6$ \\
\hline Medici & $17.8 \pm 0.4$ & $22.2 \pm 0.4$ & $9.0 \pm 0.2$ & $11.9 \pm 0.3$ & $8.8 \pm 0.3$ & $10.3 \pm 0.2$ \\
\hline
\end{tabular}

Results are expressed as mean raw scores with standard error of the mean. The maximum score was 36 for all questions, 18 for questions relating to the Medici program, and 18 for questions not relating to the program.

\section{Results}

In total, 105 students completed both the pre-tuition and the post-tuition tests. This consisted of 20 students in the control group, and 85 students in the group given access to the Medici software package. The two groups differed in their pre-tuition performance. This difference was never satisfactorily explained. The post-tuition test score of the control group was not significantly higher than their pre-tuition test score $(p>0.05)$. The computer group showed a statistically significant increase in mean total score of $12.4 \%$ over the control group $(p<0.0001)$. This increase consisted of a $15.6 \%$ improvement where the assessment questions were related to the computer material $(p<0.0001)$ and a 9.2\% increase in score for noncomputer-related questions $(p=0.0015)$ (Tables 1,2$)$.

These increases in test score remained highly statistically significant after two test questions relating to paediatric ophthalmology, which was taught in the lecture, were excluded from the analysis.

Formal monitoring of Medici usage showed that each student attempted an average of 14 of the 21 available cases $(67 \%)$, the total number ranging from one to 21 . Total time spent on the cases ranged from $7 \mathrm{~min}$ to $12 \mathrm{~h}$ $16 \mathrm{~min}$. The bulk of this time was spent on the first approach through the case, but $49 \%$ of the students attempted each case on at least two occasions, and a further $16 \%$ made additional attempts on the cases. Informal observation of the study habits of these students in the computer suite showed that they often worked in pairs with textbooks at hand. The computer material was not rushed through, but each stage of case management was discussed thoroughly.

\section{Discussion}

Over the last decade there has been a strong tendency to promote computer-based instruction as a learning medium. However, the underlying motive may be that teacher wishes to keep up with educational fashion, rather than the computer offering a true educational advantage. ${ }^{12}$ In addition, a significant amount of effort is required to create this teaching aid. Construction of the
Medici software package entailed significant programming effort, and a group of ophthalmologists were required to write and revise the case studies, under the supervision of a dedicated editor. For these reasons, the new resource must be shown to be effective.

Effectiveness in medical tuition can be measured in a number of ways, including increase in medical knowledge or clinical reasoning, improvement in clinical competence and stimulation of further learning. The present study has satisfied at least two of these criteria. Using appropriate computer-aided learning tools as an adjunct to conventional teaching regimes, we have shown that ophthalmic knowledge can be increased, and that the resource can have a positive impact on student learning in this medical discipline.

We have previously shown that computer-aided tools can be used to enhance student learning. ${ }^{11}$ Bearing this in mind, it is not surprising that students tested on material covered by the Medici computer program should show an improvement in ophthalmic knowledge. An unexpected finding was the improvement in performance in areas the computer material did not cover. There are a number of possible explanations for this finding. Firstly, the computer material was designed such that information was hidden in the case-based scenarios, rather than relayed in a purely didactic fashion. This style of delivery is intended to foster a student's interest, and stimulate further study. Secondly, the problem-based nature of the computer material may have encouraged group discussion, which might have led to a broadening of the students' knowledge base. Our informal observations of student study habits in the computer suite were consistent with these explanations. The limited extra tuition provided by the two lectures and the single attendance at the outpatient clinic may also have acted as a stimulus for learning in other areas.

The overall marks in the pre- and post-tuition assessments were relatively low, but perhaps this is not surprising considering that participation in the study was voluntary and the results of the tests had no bearing on overall student assessment. There is an apparent difference in the pre-tuition scores of the two groups. We are unable to provide any explanation for this difference

Table 2. Differences in post-tuition test scores between students given access to the Medici software package and control students who did not use the program

\begin{tabular}{ccc}
\hline Test questions & Score difference & $p$ value \\
\hline All questions & $12.4 \pm 2.9$ & $<0.0001$ \\
Medici-related questions & $15.6 \pm 2.9$ & $<0.0001$ \\
Non-related questions & $9.2 \pm 3.6$ & 0.0015 \\
\hline
\end{tabular}

Results are expressed as percentage differences with standard error of the mean. 
and accept that this might be a type II statistical error. It should be noted that this does not affect the statistics on which the study is based.

The medical students improved their test score by $19 \%$ when examined on material covered by the computer case studies. Should this be considered adequate justification for replacing didactic lectures and tutorials with computer-aided instruction? Our study does not provide information about the relative value of a lecture series in teaching ophthalmology. Current educational philosophy certainly suggests that students actively participating in an interactive computer experience will be more stimulated than those attending a passive lecture course. Further, some studies indicate that well-designed material on a computer is of relatively greater benefit. ${ }^{13,14}$ However, whilst computer-aided learning may be preferable to more traditional teaching methods, the primary purpose of the clinical teaching program remains unchanged, that is, to focus medical students on a particular discipline. In this study, students had 2 weeks specifically to explore ophthalmology. They had access to the outpatient clinic, clinical specialists in the field and the medical library, as well as the Medici software package. A combination of all these resources is probably necessary to maximise the students' educational experience. It is unreasonable to expect a computer program to teach students how to use a direct ophthalmoscope competently, just as it is unreasonable to expect a student to see the entire range of ophthalmic conditions during a single visit to the outpatient clinic.

In conclusion, there are several applications for computer-aided instruction in the tuition of ophthalmology. Visual pattern recognition is an important part of ophthalmic diagnosis, and management frequently involves one or more procedures. Consequently, this discipline is ideally suited to teaching with multimedia methods. In this study, we have demonstrated a useful role for an appropriately designed computer software program in imparting new knowledge to medical students. The program also had a positive impact on student learning in the field in general. Teaching of clinical examination and procedural skills may be supplemented by this resource. For example, a software program instructing on assessment of pupillary light responses reduced time spent by faculty members in teaching and increased student efficiency at learning the skill. ${ }^{15}$ Computer technology may also be useful in the teaching of advanced ophthalmic skills to ophthalmic trainees and practitioners. ${ }^{16}$ Although there is initial effort and expense to produce a computer-aided learning product, once created, revision of the content is relatively straightforward. This can have a significant beneficial impact on teaching resources and be a boon for students and practitioners working at hospitals located far from major teaching hospitals.

\section{References}

1. Miflin BM, Campbell CB, Price DA. A lesson from the introduction of a problem-based, graduate entry course: the effects of different views of self-direction. Med Educ 1999;33:801-7.

2. Schwartz PL, Loten EG, Miller AP. Curriculum reform at the University of Otago Medical School. Acad Med 1999;74:675-9.

3. Peters AS, Greenberger-Rosovsky R, Crowder C, Block CD, Moore GT. Long-term outcomes of the new pathway program at Harvard Medical School: a randomized controlled trial. Acad Med 2000;75:470-9.

4. O'Neill PA, Morris J, Baxter C-M. Evaluation of an integrated curriculum using problem-based learning in a clinical environment: the Manchester experience. Med Educ 2000;34:222-30.

5. Spencer JA, Jordan RK. Learner centred approaches in medical education. BMJ 1999;318:1280-3.

6. Shuttleworth GN, Marsh GW. How effective is undergraduate and postgraduate teaching in ophthalmology? Eye 1997;11:744-50.

7. Jacobs DS. Teaching doctors about the eye: trends in the education of medical students and primary care residents. Surv Ophthalmol 1998;42:383-9.

8. Farrell TA, Albanese MA, Pomrehn PR Jr. Problem-based learning in ophthalmology: a pilot program for curricular renewal. Arch Ophthalmol 1999;117:1223-6.

9. Whitcomb ME, Anderson MB. Transformation of medical students' education: work in progress and continuing challenges. Acad Med 1999;74:1076-9.

10. Devitt P, Cehic D, Palmer E. Computers in medical education. 2. Use of a computer package to supplement the clinical experience in a surgical clerkship: an objective evaluation. Aust N Z J Surg 1998;68:428-31.

11. Devitt P, Palmer E. Computers in medical education. 1. Evaluation of a problem-oriented learning package. Aust N Z J Surg 1998;68:284-7.

12. Campbell JK, Johnson C. Trend spotting: fashions in medical education. BMJ 1999;318:1272-5.

13. Khadra $\mathrm{MH}$, Guinea $\mathrm{AI}$. Interactive urology: an evaluation. Aust N Z J Surg 1996;66:478-80.

14. Andrews PV, Schwarz J, Helme RD. Students can learn medicine with computers. Med J Aust 1992;157:693-5.

15. Kaufman D, Lee S. Formative evaluation of a multimedia CAL program in an ophthalmology clerkship. Med Teacher 1993;15:327-40.

16. Sinclair MJ, Peifer JW, Haleblian R, Luxenberg MN, Green K, Hull DS. Computer-simulated eye surgery: a novel teaching method for residents and practitioners. Ophthalmology 1995;102:517-21. 